\title{
Preparação de argilas organofílicas usando diferentes concentrações de sal quaternário de amônio
}

\section{(Preparation of organophilic clays using different concentrations of quaternary ammonium salt)}

\author{
A. A. Silval, F. R. Valenzuela-Diaz ${ }^{2}$, G. S. V. Martins ${ }^{1}$, M. G. F. Rodrigues ${ }^{l}$ \\ ${ }^{I}$ Centro de Ciências e Tecnologia - CCT, Universidade Federal de Campina Grande - UFCG \\ Av. Aprígio Veloso 882, Bodocongó, Campina Grande, PB 58109-970 \\ ${ }^{2}$ Departamento de Engenharia Metalúrgica e Materiais, Escola Politécnica da Universidade de S. Paulo \\ meiry@deq.ufcg.edu.br
}

\begin{abstract}
Resumo
Estudou-se a preparação de argilas organofílicas com o sal cloreto hexadeciltrimetilamônio (HDTMA-Cl) a diferentes concentrações. As amostras sem e com tratamento com o sal HDTMA-Cl foram caracterizadas por difração de raios X, análise térmica diferencial, análise termogravimétrica, microscopia eletrônica de varredura e espectroscopia de raios X por energia dispersiva. Ensaios de difração de raios X mostraram que a distância basal da argila "Chocolate" antes da modificação era 14,8 e após tratamento com o sal HDTMA-Cl (0,8 meq/g) é 19,1 e com o sal HDTMA-Cl (1,0 meq/g) 17,8 . O processo de intercalação não causou modificações substanciais na morfologia das partículas da argila "Chocolate".

Palavras-chave: argila, argila organofilica, HDTMA-Cl.
\end{abstract}

\section{Abstract}

The preparation of organophilic clays using the salt hexadecyltrimethyl ammonium chloride (HDTMA-Cl) at different concentrations was studied. The samples before and after treatment with HDTMA-Cl salt were characterized by X-ray diffraction, differential thermal analysis, thermogravimetric analysis, scanning electron microscopy and X-ray energy dispersive spectroscopy. X-ray diffraction analysis show that the basal spacing of the clay "Chocolate" before the modification was 14.8 and after treatment with the HDTMA-Cl salt (0,8 meq/g) 19.1 and with HDTMA-CL (1,0 meq/g) 17.8. The intercalation process did not cause substantial modifications in the morphology of particles of the "Chocolate" clay.

Keywords: clay, organoclays, HDTMA-Cl.

\section{INTRODUÇÃO}

As esmectitas são largamente usadas numa gama de aplicações devido a sua capacidade de troca de cátions, capacidade de expansão, alta área específica e por possuírem fortes capacidades de adsorção/absorção [1-4]. Dentro das argilas que tem a capacidade de expandir, a mais comum esmectita dioctaédrica é a montmorilonita.

A montmorilonita possui uma carga negativa na superfície que pode ser substituída isomorficamente. Ela é, naturalmente, balanceada pelos cátions inorgânicos trocáveis, tais como $\mathrm{Na}^{+}$ e $\mathrm{Ca}^{+2}$, que são geralmente hidratados na presença de água. A hidratação dos cátions trocáveis e a natureza polar dos grupos silanois ( $\mathrm{Si}-\mathrm{O}$ ) conferem uma natureza hidrofílica a superfície da montmorilonita, o que torna essas argilas naturais solventes ineficazes para compostos orgânicos [5-7].

Como os íons metais são substituídos por cátions surfactantes, tais como íons alquilamônio, a natureza da superfície é drasticamente modificada. Enquanto as argilas naturais são geralmente hidrofílicas, as argilas modificadas com os surfactantes (organofílicas) são hidrofóbicas ou organofílicas [6-9]. As argilas organofílicas são facilmente solvatadas e expandidas por vários solventes orgânicos, o que torna as argilas organofílicas atrativas para adsorventes seletivos, agentes gelatinosos de sistemas orgânicos, etc.

Diversas argilas organofílicas tem sido preparadas e caracterizadas, a partir de argilas esmectíticas brasileiras [10-12]. Em estudo sobre a obtenção de argila organofílica com argila esmectítica policatiônica e sal quaternário Arquad 2HT-75, foi verificada a intercalação das moléculas do sal quaternário entre as lâminas do argilomineral, após apresentar distância basal superior a $3 \mathrm{~nm}$ [11]. Após preparação de argilas organofílicas, a partir de bentonitas de Campina Grande-PB e do sal quaternário cloreto de cetil trimetil amônio e estudo de inchamento em etanol, tolueno e diesel, foi observado por meio de difração de raios $\mathrm{X}$ a efetiva 
intercalação dos cátions quaternários de amônio entre os espaços interlamelares dos argilominerais. Os autores concluem ainda, que para os solventes utilizados nesse estudo, o inchamento é inferior aos obtidos anteriormente com outras argilas do Estado da Paraíba [13]. Foi feito o estudo sobre a influência da concentração de argila (de 0,5 a 6,0\%), nas dispersões aquosas de partida, para obtenção de argilas organofílicas e estudo de inchamento em três solventes [14]; o material obtido desta preparação apresentou distâncias interlamelares superiores a $3 \mathrm{~nm}$.Foi também verificado que os materiais que apresentaram maiores valores de inchamento, obtidos usando concentrações inicias de argila entre 1,0 e 3,0\%.

Ultimamente as argilas organofílicas vêm ganhando notoriedade por estarem sendo utilizadas na obtenção de nanocompósitos bentonitas/polímeros, um novo tipo de material com amplo potencial de uso, principalmente nas indústrias automotivas e de embalagens [15]. Este trabalho faz parte de uma série que vem pesquisando a obtenção e caracterização de argilas organofílicas, visando seu uso na indústria petrolífera, no tratamento de substâncias contaminadas e na obtenção de nanocompósitos silicato/ polímeros. Levando-se em consideração que o Estado da Paraíba possui as maiores reservas brasileiras de argilas esmectíticas, este estudo foi realizado para obter e caracterizar uma argila "Chocolate" nacional modificada com o sal cloreto hexadeciltrimetilamônio (Genamim CTAC-50) visando sua utilização num sistema de separação óleo/água.

\section{EXPERIMENTAL}

Utilizou-se na preparação de argilas bentoníticas organofílicas, a bentonita policatiônica "Chocolate", fornecida pela empresa Dolomil Ind. Ltda. na sua forma bruta, peneiradas em peneira ABNT 200 (0,074 mm), sem modificação das propriedades químicas. Utilizou-se o sal quaternário de amônio comercial brasileiro denominado cloreto de hexadecil Trimetil amônio (Genamim-CTAC50) com duas relações argila/sal: 80 meq/100 g de argila e $100 \mathrm{meq} / 100 \mathrm{~g}$ de argila, respectivamente [16]. Especificações do sal (Genamim-CTAC50): Solução em etanol a $50 \%$, fórmula $94 \%$ de $\mathrm{C} 16,5 \%$ de $\mathrm{C} 14$ e $1 \%$ de $\mathrm{C} 12$, fornecedor Clariant.

Para a preparação da argila organofílica seguiu-se duas etapas: na primeira, a argila chocolate policatiônica foi transformada para a forma sódica utilizando uma dispersão aquosa com concentração de $4 \%$ em peso de argila desagregada e moída. Acrescentou-se à dispersão aquosa solução de carbonato de sódio concentrado na proporção de $100 \mathrm{meq} /$ $100 \mathrm{~g}$ de argila seca, sob agitação constante e aquecimento até $95{ }^{\circ} \mathrm{C}$ por 30 minutos; na segunda etapa, adicionou-se, a solução de sal quaternário de amônio GENAMIN CTAC-50 a $25 \%$, e após adição completa do sal, agitou-se por $30 \mathrm{~min}$ para filtração a vácuo em seguida. Os sólidos obtidos foram colocados em estufa a $60 \pm 5{ }^{\circ} \mathrm{C}$ por $48 \mathrm{~h}$, e desagregados e peneirados em peneira ABNT $200(0,074 \mathrm{~mm})$.

As amostras, após tratamento com o sal quaternário de amônio são denominadas da seguintes forma: as argilas que foram tratadas com o sal Genamim-CTAC50 com a relação
1,0 meq/100 g de argila são denominadas argila $100 \mathrm{G}(100$ indica a relação argila/sal e o $G$ indica o sal GenamimCTAC50), por exemplo Chocolate 100G; as tratadas com o sal Genamim-CTAC50 com a relação $0,8 \mathrm{meq} / 100 \mathrm{~g}$ de argila são argila $80 \mathrm{G}$, por exemplo Chocolate $80 \mathrm{G}$.

As amostras de argilas policatiônicas, sódica e modificadas com o sal quaternário de amônio foram submetidas às seguintes técnicas de caracterização:

Difração de raios $X(D R X)$ - difratômetroShimadzu XDR 6000 com radiação $\mathrm{k}_{\alpha}$ do cobre, com tensão $40 \mathrm{kV}$, corrente $40 \mathrm{~mA}$, passo $0,02 \mathrm{em} 2 \theta$ e tempo por passo de $1 \mathrm{~s}$. O objetivo desta técnica é verificar a existência de minerais acessórios (quartzo, feldspato) e de argilominerais.

Análise térmica (ATD/TG) - equipamento Shimadzu, com vazão de $100 \mathrm{~mL} / \mathrm{min}$ de ar desde temperatura ambiente até $1000{ }^{\circ} \mathrm{C}$, com taxa de aquecimento $10^{\circ} \mathrm{C} / \mathrm{min}$. Alfaalumina foi utilizada como material de referência. Estas técnicas permitem avaliar a temperatura de modificação e decomposição do sal organoclorado no material.

Microscopia eletrônica de varredura (MEV) - A amostra na forma de pó foi fixada no porta-amostra com fita adesiva de carbono e recoberta com uma fina camada de ouro num metalizador. As micrografias para análise da morfologia das amostras foram obtidas no microscópio eletrônico de varredura Philips XL30 equipado com o sistema de espectroscopia de raios $\mathrm{X}$ por energia dispersiva (EDS). Esta técnica é indicada para o estudo da morfologia dos cristais individuais dos minerais argilosos, bem como para a análise química elementar.

\section{RESULTADOS E DISCUSSÃO}

Com a troca do sódio pelo cátion do sal quaternário HDTMA-Cl, ocorre a expansão da camada da montmorilonita. Esta expansão é detectada por difração de

\section{E: pico característico da esmectita}

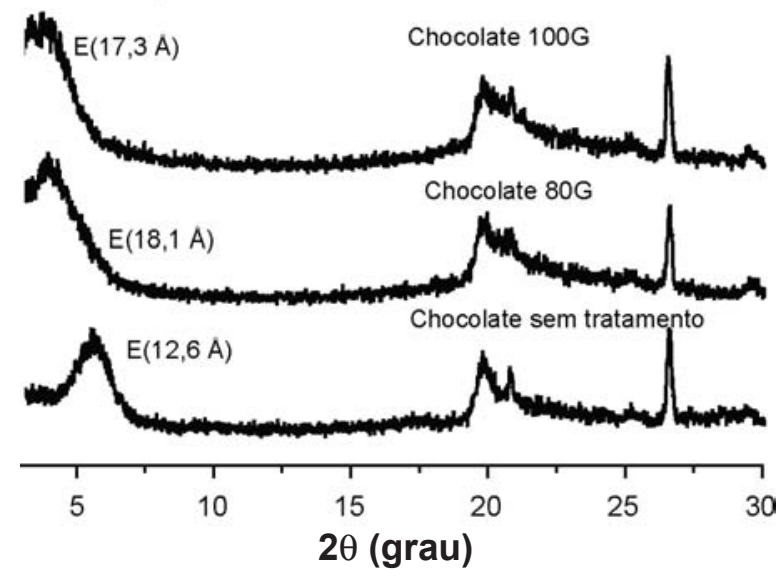

Figura 1: Curvas de difração de raios X da argila "Chocolate" sem tratamento e modificada com o sal HDTMA-Cl a diferentes concentrações.

[Figure 1: X-ray diffraction patterns of the "Chocolate" clay

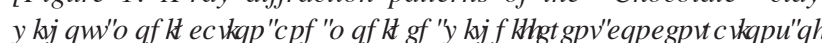
HDTMA-C1 salt.] 

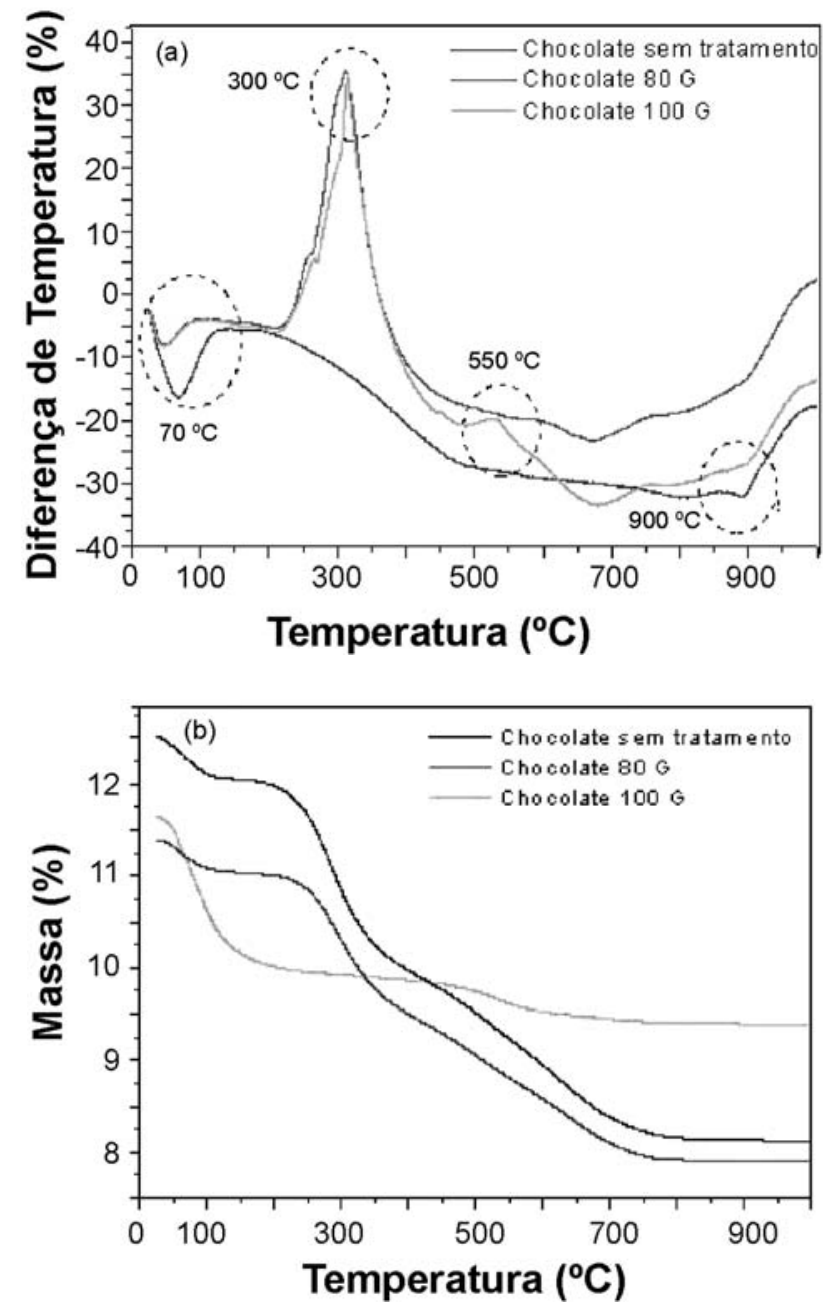

Figura 2: Curvas de análises térmicas, (a)ATD e (b)TG, da argila "Chocolate" sem tratamento e com tratamento com o sal HDTMA$\mathrm{Cl}$ a diferentes concentrações.

[Figure 2: DTA (a) and TG (b) curves of the "Chocolate" clay ZLWRXWP RGÀFDURQDQGP RGÀHGZ LK GIIHHQMFRQFHQWDURQVRI HDTMA-C1 salt.]

raios X [17]. A curva de difração da argila "Chocolate" sem tratamento está na Fig. 1. A amostra apresenta reflexão do grupo da esmectita que corresponde à distância basal $\left(\mathrm{d}_{001}\right)$ 12,6 Å para a argila "Chocolate" sem tratamento. Observase também o pico que corresponde ao quartzo (Q) [18-20]. Quando a argila "Chocolate" é tratada com o sal HDTMA$\mathrm{Cl}$ ocorrem modificações nos espaçamentos basais da argila. Na Fig. 1 são apresentadas as curvas de difração da argila "Chocolate" sem tratamento e esmectítica "Chocolate" com o surfactante a diferentes concentrações. A argila "Chocolate" apresenta originalmente uma distância basal de 12,6 ̊̊ que é modificada com a incorporação do HDTMA-Cl para o valor de $18,1 \AA$, quando a proporção do HDTMA-Cl é de $0,8 \mathrm{meq} / \mathrm{g}$ e $17,3 \AA$ quando a proporção do HDTMA-Cl é de $1,0 \mathrm{meq} / \mathrm{g}$. Estes resultados confirmam a hipótese de que o HDTMA-Cl foi intercalado nos espaços lamelares da argila "Chocolate" [18-21].
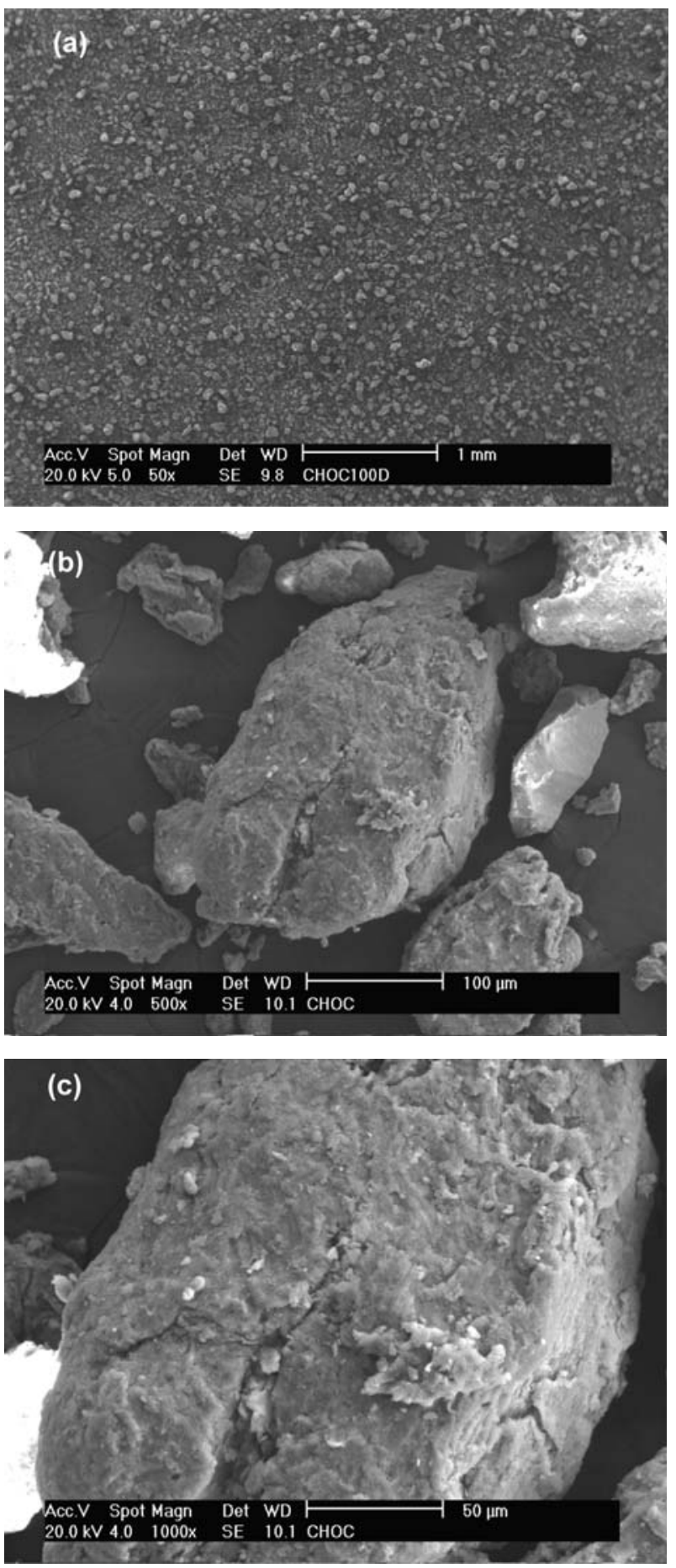

Figura 3: Micrografias eletrônicas de varreduras da argila "Chocolate" sem tratamento.

[Figure 3: SEM micrographs of the "Chocolate" clay]

Na Fig. 2 são mostradas as curvas TG e ATD da argila "Chocolate" sem tratamento e com tratamento.

A análise térmica diferencial para a argila "Chocolate" sem tratamento na Fig. 2a exibe um pico endotérmico entre $30{ }^{\circ} \mathrm{C}$ e $140{ }^{\circ} \mathrm{C}$ referente à perda de água livre, e outro pico endotérmico entre $490{ }^{\circ} \mathrm{C}$ e $550{ }^{\circ} \mathrm{C}$ correspondente à 

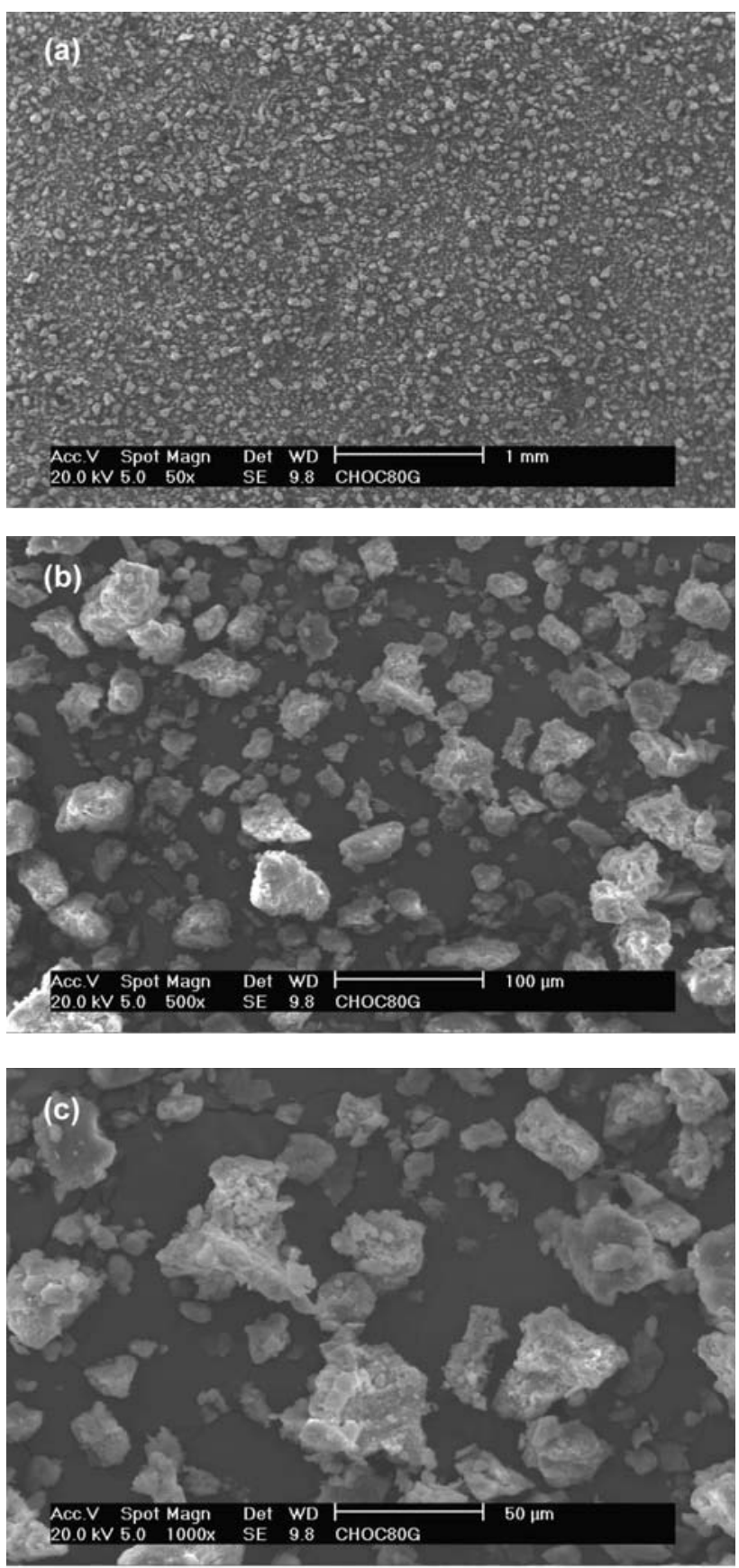

Figura 4: Micrografias eletrônicas de varreduras da argila "Chocolate" modificada com o sal HDTMA-Cl na proporção $0,8 \mathrm{meq} / \mathrm{g}$ de argila.

>) IJXUH 6I O PIFLRJUSKV RI WKH' \&KRFRФUAL FQ PR PGÀHG with HDTMA-C1 salt, 0.8 meq/g of clay.]

deshidroxilixação do argilomineral. O pico exotérmico a $850{ }^{\circ} \mathrm{C}$ é estrutural característico da destruição do reticulado cristalino. A curva de análise térmica diferencial obtida para a argila "Chocolate" tratada com o sal HDTMA-Cl, com concentração de $0,8 \mathrm{meq} / \mathrm{g}$, mostra um pico endotérmico na faixa de $20^{\circ} \mathrm{C}$ a $100{ }^{\circ} \mathrm{C}$ atribuído à perda de água livre e um pico exotérmico que ocorre entre $200^{\circ} \mathrm{C} \mathrm{e} 400^{\circ} \mathrm{C}$ atribuído à
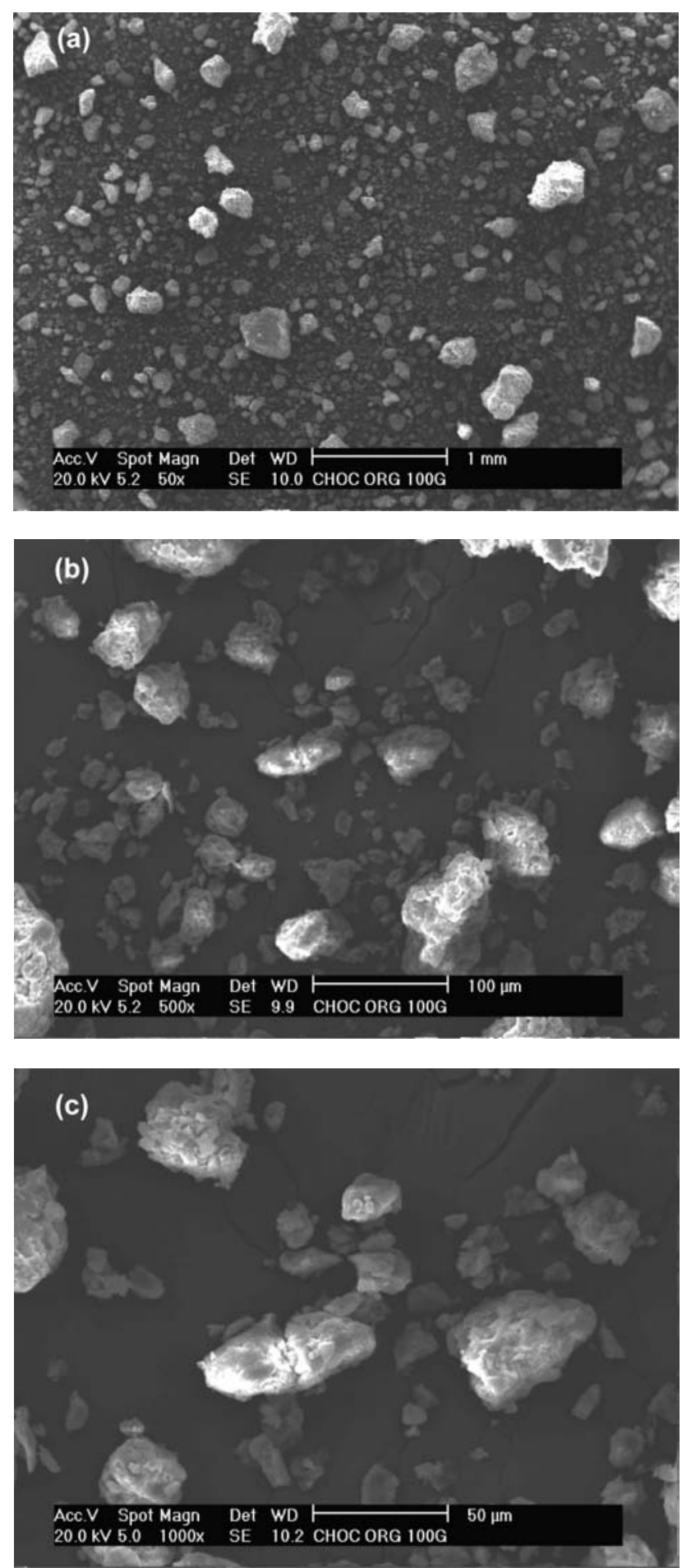

Figura 5: Micrografias eletrônicas de varreduras da argila "Chocolate" modificada com o sal HDTMA-Cl na proporção $1,0 \mathrm{meq} / \mathrm{g}$ de argila.

>) IJXUH 6( O PIFURJUSKV RI UKH' \&KRFRФUA FO PRGÀHG with HDTMA-C1 salt, $1.0 \mathrm{meq} / \mathrm{g}$ of clay.]

decomposição inicial do sal quaternário de amônio HDTMACl, após a etapa de liberação da água residual e combustão da parte orgânica proveniente do sal quaternário de amônio. $\mathrm{Na}$ faixa entre $500{ }^{\circ} \mathrm{C}$ e $800{ }^{\circ} \mathrm{C}$ há dois picos superpostos, 
exo-endotérmico; o primeiro deve-se à decomposição final do sal orgânico e o segundo à combustão do resíduo carbonoso da queima anterior, caracterizada pelo largo pico de ATD exotérmico [22]. Na análise termogravimétrica a argila "Chocolate" sem tratamento e modificada, com o sal quaternário HDTMA-Cl está apresentada na Fig. 2b. O teor de água livre perdida é bem maior para a argila "Chocolate" sem tratamento (aproximadamente 12,5\%). Para a argila "Chocolate" $80 \mathrm{G}$ a perda de massa é de aproximadamente 11,4\% e para a argila "Chocolate" $100 \mathrm{G}$ é de aproximadamente $11,7 \%$. Podemos observar claramente que com o tratamento das argilas com o sal, a perda de massa evidencia que o caráter hidrofílico da argila foi reduzido. De modo geral, o comportamento das curvas de TG apresentam tendências negativas; isto se deve ao fato de que a queima dos radicais orgânicos fazem a amostra diminuir a massa e, portanto, há uma variação negativa da massa na curva de TG.
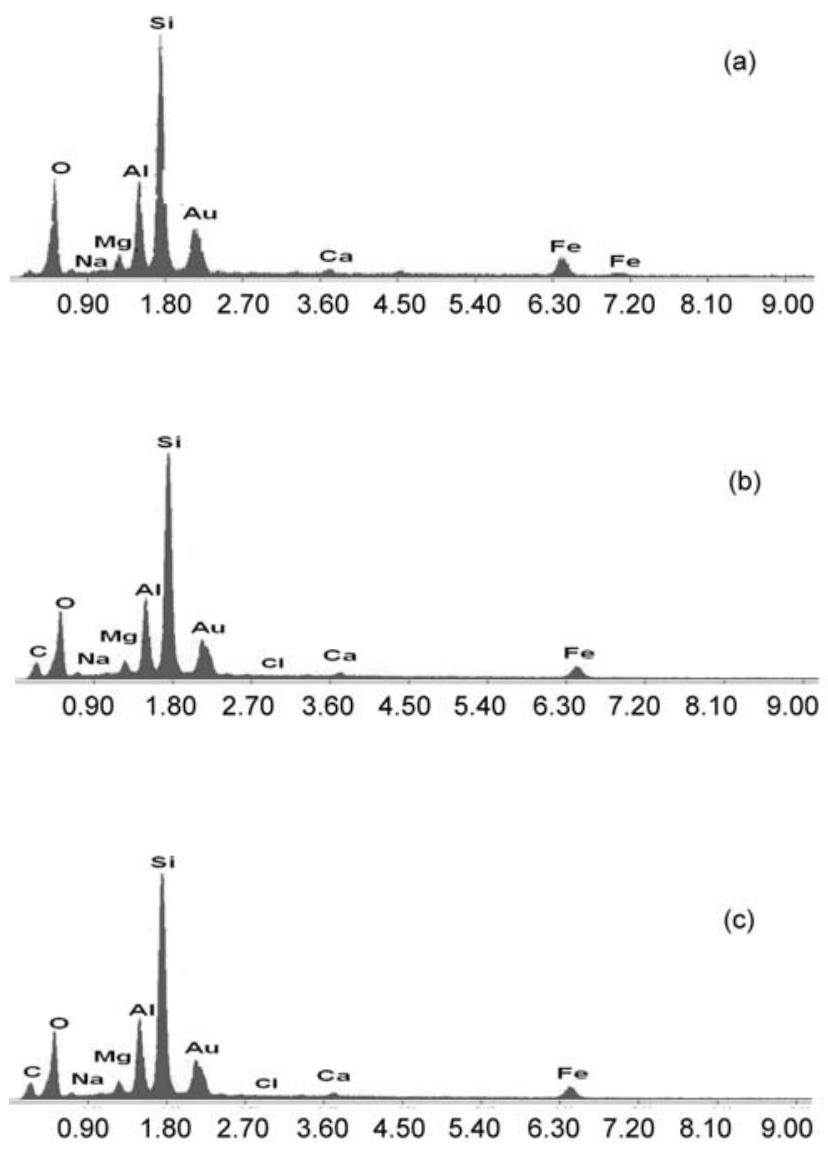

Figura 6: Espectroscopia de raios $\mathrm{X}$ por energia dispersiva das argila "Chocolate": (a) sem tratamento, (b) com tratamento com o sal HDTMA-Cl na concentração de $0,8 \mathrm{meq} / \mathrm{g}$ de argila e (c) com tratamento com o sal HDTMA-Cl na proporção de 1,0meq/g de argila.

[Figure 6: EDS microanalysis spectrum of the clay "Chocolate". (a) without treatment, (b) with treatment with salt HDTMA-Cl in the ratio of $0,8 m e q / g$ of clay and (c) with treatment salt HDTMA-Cl in the ratio of $1,0 m e q / g$ of clay.]
Nas Figs. 3 a 5 são apresentadas as micrografias eletrônicas de varredura das amostras "Chocolate" sem e com tratamento com o sal HDTMA-Cl. Uma argila lamelar seca pode ser descrita por três unidades estruturais e pelo arranjo dessas unidades no espaço [26]. De forma geral, a partir das observações das micrografias eletrônicas de varredura (Figs. 3 a 5) é possível verificar que todas as amostras de argila "Chocolate" com e sem tratamento apresentam morfologia similar. Não há uma distribuição muito homogênea de partículas pois apresentam grãos de diversos tamanhos, ocasionando, portanto, uma distribuição de partículas irregular. Essas amostras são constituídas por aglomerados de partículas irregulares.

A Fig. 6 apresenta os resultados obtidos para a argila "Chocolate": (a) sem tratamento, (b) modificada com o sal HDTMA-Cl com concentração de 0,8 meq $/ \mathrm{g}$ e (c) modificada com o sal HDTMA-Cl com concentração de 1,0meq/g.

Verificou-se que a amostra "Chocolate" sem tratamento apresenta em sua composição, basicamente, Si e Al, característico de argilas esmectíticas, além da presença de $\mathrm{Na}, \mathrm{Mg}, \mathrm{Ca}$ e Fe. Nas argilas "Chocolate" com tratamento com HDTMA-Cl, independente da concentração $(0,8 \mathrm{meq} / \mathrm{g}$ e $1,0 \mathrm{meq} / \mathrm{g}$ ) é possível identificar qualitativamente os mesmos compostos presentes na argila sem tratamento, além do $\mathrm{Cl}$ e C, provenientes da composição do sal HDTMA-Cl.

\section{CONCLUSÕES}

As mudanças na distância basal sugerem a formação de bicamadas de sais quaternários (HDTMA-Cl) nos espaços interlamelares. A argila "Chocolate" sem tratamento apresenta uma distância basal de $12,6 \AA ̊$ e é modificada com a incorporação do HDTMA-Cl; o valor da distância basal aumenta para $18,1 \AA ̊$ quando a concentração do HDTMA-Clé 0,8meq/g (80G) e 17,3 $\AA$ quando a concentração do HDTMAClé 1,0meq/g (100G).Apresença do sal quaternário de amônio (HDTMA-Cl) nos espaços interlamelares foi confirmada por difração de raios X. O processo de intercalação não causou modificações substanciais na morfologia das partículas da argila "Chocolate".

\section{AGRADECIMENTOS}

Os autores deste trabalho agradecem, em especial, ao PRH-25/ANP/MCT pelo auxílio de bolsa de mestrado.

\section{REFERÊNCIAS}

[1] G. R. Alther, Contam. Soils 8 (2003) 189.

[2] G. R. Alther, Special Publication - Royal Society of Chemistry 259 (2000) 277.

[3] C. Breen, R. Watson, J. Madejova, P. Komadel, Z. Klapyta, Langmuir 13 (1997) 6473.

[4] S. K. Dentel, J. Y. Bottero, K. Khatib, H. Demougeot, J. P. Duguet, C. Anselme, Water Research 29 (1995) 1353.

[5] J. F. Lee, M. M. Mortland, C. T. Chiou, S. A Boyd, Faraday Trans. 1 (1985) 2953. 
[6] T. Permien, G. Lagaly, Colloid Polym. Sci. 272 (1994) 1306.

[7] M. M. Mortland, S. Sun, S. A. Boyd, Clays Clay Minerals 38 (1990) 113.

[8] S. A. Boyd, M. M. Mortland, C. T. Chiou, Soil - Science Society Am. J. 52 (1988) 658.

[9] S. A. Boyd, S. Sun, J. F. Lee, M. M. Mortiand, Clays Clay Minerals 36 (1988) 125.

[10] F. R. Valenzuela-Diaz, Tese de Doutorado, Escola Politécnica da Universidade de S. Paulo, S. Paulo (1994).

[11] F. R. Valenzuela-Diaz, Key Eng. Mater. 189-191 (2001) 203.

[12] F. R. Valenzuela-Diaz, W. S. B. Mourão, A. C. V. Coelho, P. Souza Santos, Anais $12^{\circ}$ Cong. Bras. Eng. Quím. (cd-rom) Porto Alegre, RS (1998).

[13] A. A. Silva, V. F. J. Kozievitch, C. L. V. José, S. M. Toffoli, M. G. F. Rodrigues, H. L. Lira, H. C Ferreira, F. R. Valenzuela-Diaz, Anais $46^{\circ}$ Cong, Bras, Ceram. (cd-rom), S. Paulo, SP (2002).
[14] F. R. Valenzuela-Diaz, Anais $43^{\circ}$ Cong. Bras. Ceram. (cd-rom), Florianópolis, SC (1999).

[15] T. J. Pinnavaia, Polymer-Clay Nanocomposites, John Wiley, New York (2001).

[16] K. R. O. Pereira, R. A. Hanna, M. M. G. R. Vianna, C. A. Pinto, M. G. F. Rodrigues, F. R. Valenzuela-Diaz, Mater. Res. 8 (2005) 1.

[17] Y. Xi, Z. Ding, H. He, R. L. Frost,- J. Colloid Interface Sci. 277 (2004) 116.

[18] C. C. Wang, L. C. Juang, C. K. Lee, T. C. Hsu, J. F. Lee, H. P. Chao, J. Colloid Interface Sci. 280 (2004) 27.

[19] Y. H. Shem, Chemosphere 44 (2001) 989.

[20] J. H. Choy, S. Y. Kwak, Y. S. Han, B. W. Kim, Mater. Lett. 33 (1997) 143.

[21] M. Kozak, L. Domda, J. Phys. Chem. Solids 65 (2004) 441.

[22] G. Lagaly, Clays Clay Minerals 30 (1982) 215.

(Rec. 31/12/2005, Rev. 04/05/2006, 17/08/2007, Ac. 24/08/2007) 